\title{
Endoscopic epicardial unipolar ablation - a viable approach?
}

\author{
Cristian Dumitrescu ${ }^{1}$, Kerstin Bauer ${ }^{1}$, Juraj Melichercik ${ }^{1}$, Ralf Sodian ${ }^{2}$, Eberhard von \\ Hodenberg, ${ }^{1}$, and Stefan Bauer ${ }^{1}$ \\ ${ }^{1}$ MediClin Herzzentrum Lahr/Baden \\ ${ }^{2}$ MediClin Heart Centre Lahr/Baden
}

October 26, 2020

\begin{abstract}
The treatment of persistent atrial fibrillation (PSAF) remains a challenge for electrophysiologists. Nowadays, isolation of the pulmonary veins (PVI) is a routinely applied, well proven and accepted intervention. Despite this, some patients are in refractory atrial fibrillation (AF) even after 2 or more procedures. Others are unable to receive another catheter ablation due to previous cardiac interventions. The Convergent procedure might be a good option for this type of patients. Here we report on two of these cases from our clinic: one of a female patient who underwent a TAVI procedure in our institution 3 months before the epicardial Ablation and the second old male patient with paroxysmal AF who underwent percutaneous transcatheter closure of patent foramen ovale (PFO) in 2015 after having an embolic stroke. These two cases show that the staged Convergent procedure can be performed safely and with good results even in patient who underwent previous cardiac interventions. In addition, it might be a good alternative in patients in whom a primary transcatheter ablation is impossible due to previous pathologies and interventions.
\end{abstract}

\section{Endoscopic epicardial unipolar ablation - a viable approach?}

Authors Dumitrescu, Cristian MD 1 ; Bauer, Kerstin MD 1; Melichercik, Juraj MD 2; Sodian, Ralf MD 1; von Hodenberg, Eberhard MD 2; Bauer, Stefan MD 1

Affiliation: 1 Department of Cardiac Surgery, Herzzentrum Lahr, D-77933 Lahr 2 Department of Cardiology, Herzzentrum Lahr, D-77933 Lahr

Disclosure: None Funding: None

Corresponding Author Dumitrescu, Cristian MD Cardiac Surgery, Herzzentrum Lahr, D-77933 Lahr Cristian.Dumitrescu@mediclin.de cristiand_82@yahoo.com

\section{Case report of two challenging cases}

The treatment of persistent atrial fibrillation (PSAF) remains a challenge for electrophysiologists. Nowadays, isolation of the pulmonary veins (PVI) is a routinely applied, well proven and accepted intervention. Despite this, some patients are in refractory atrial fibrillation (AF) even after 2 or more procedures. Others are unable to receive another catheter ablation due to previous cardiac interventions. The Convergent procedure or the endoscopic epicardial unipolar ablation as stand-alone might be a good option for this type of patients. The Convergent procedure is a hybrid AF ablation procedure in which the surgeon via subxiphoid access performs a set of lesions on the posterior wall of the left atrium1. Then, a cardiac electrophysiologist maps and completes isolation of the pulmonary veins and posterior wall. This combined procedure can be performed in a single stage (same day) setting, or in a staged setting.

Here we report on two of these cases from our clinic: 
The first case is a 78 years old female patient with long standing persistent AF who underwent a TAVI procedure in our institution 3 months before the epicardial ablation.

The patient had undergone one pharmacologic cardioversion in the past, and was in NYHA class III with an LVEF of $35 \%$. The Convergent procedure was performed in a staged setting, with 4 months between the epicardial and the endocardial procedure. The epicardial ablation catheter (EPi-Sense Coagulation Device, AtriCure, Inc.) (3 cm ablation coil and is approximately $1 \mathrm{~cm}$ wide) was easily inserted via a subxiphoidal approach, advanced and 19 ablation lines were placed under endoscopic visualization. In order to avoid esophageal overheating, saline at room temperature was infused into the pericardial space during epicardial ablations, and the esophageal temperature was monitored with temperature probe. Upon completion of the lesion set, a pericardial drain was left in place. To complete the procedure, a transthoracic electrical cardioversion was successfully performed. A post-operative transthoracic echocardiogram (TTE) showed a fully functional nitinol aortic valve prosthesis, unaffected by the temporary rise of intrapericardial temperature. After a short episode of spontaneously terminating AF during the stay on the normal ward, the patient was discharged home in sinus rhythm (SR) on the ninth postoperative day. The patient presented back to the clinic 3 months later in sinus rhythm, and underwent the second stage of the hybrid procedure. This procedure consisted of 3D-mapping with NAVx (Endocardial Solutions, St. Jude Medical, Inc., St. Paul, MN, USA) and pulmonary vein isolation and gap closing. After this procedure, the patient left the hospital 3 days later and was discharged while still in SR showing a improved ejection fraction of over $50 \%$

The second case is a 63 years old male patient with paroxysmal AF who underwent percutaneous transcatheter closure of patent foramen ovale (PFO) in 2015 after having an embolic stroke. The patient presented to the clinic with vertigo and arm accentuated hemiparesis. Because of the PFO closure procedure the patient was not suitable for a conventional transcatheter PVI approach. Therefore, only epicardial ablation appeared to be a feasible option. The patient was in SR before the procedure. The intervention was performed the same way as described above, and in this case 23 ablation lines were placed. No intraprocedural complications occurred, and no post ablation transthoracic electrical cardioversion was necessary. On second postoperative day a transthoracic echo was performed to evaluate the PFO. The echo showed no opening between the right and left atrium and the closure device was in perfectly in place despite the temporary temperature rise and forces applied to the left atrium throughout the surgical procedure. Because of the PFO closure device, no 3D-mapping was possible. The patient was discharged in SR from the hospital

These two cases show that the endoscopic epicardial unipolar ablation as a part of staged Convergent procedure or stand-alone can be performed safely and with good results even in patient who underwent previous cardiac interventions. In addition, it might be a good alternative in patients in whom a primary transcatheter ablation is impossible due to previous pathologies and interventions.

Lawrence S. Lee. Subxiphoid Minimally Invasive Epicardial Ablation (Convergent Procedure) With Left Thoracoscopic Closure of the Left Atrial Appendage. Operative Techniques in Thoracic and Cardiovascular Surgery, 23, 152-165, 2018

\section{Hosted file}

PFO ocluder.avi available at https://authorea.com/users/370275/articles/488963-endoscopicepicardial-unipolar-ablation-a-viable-approach 


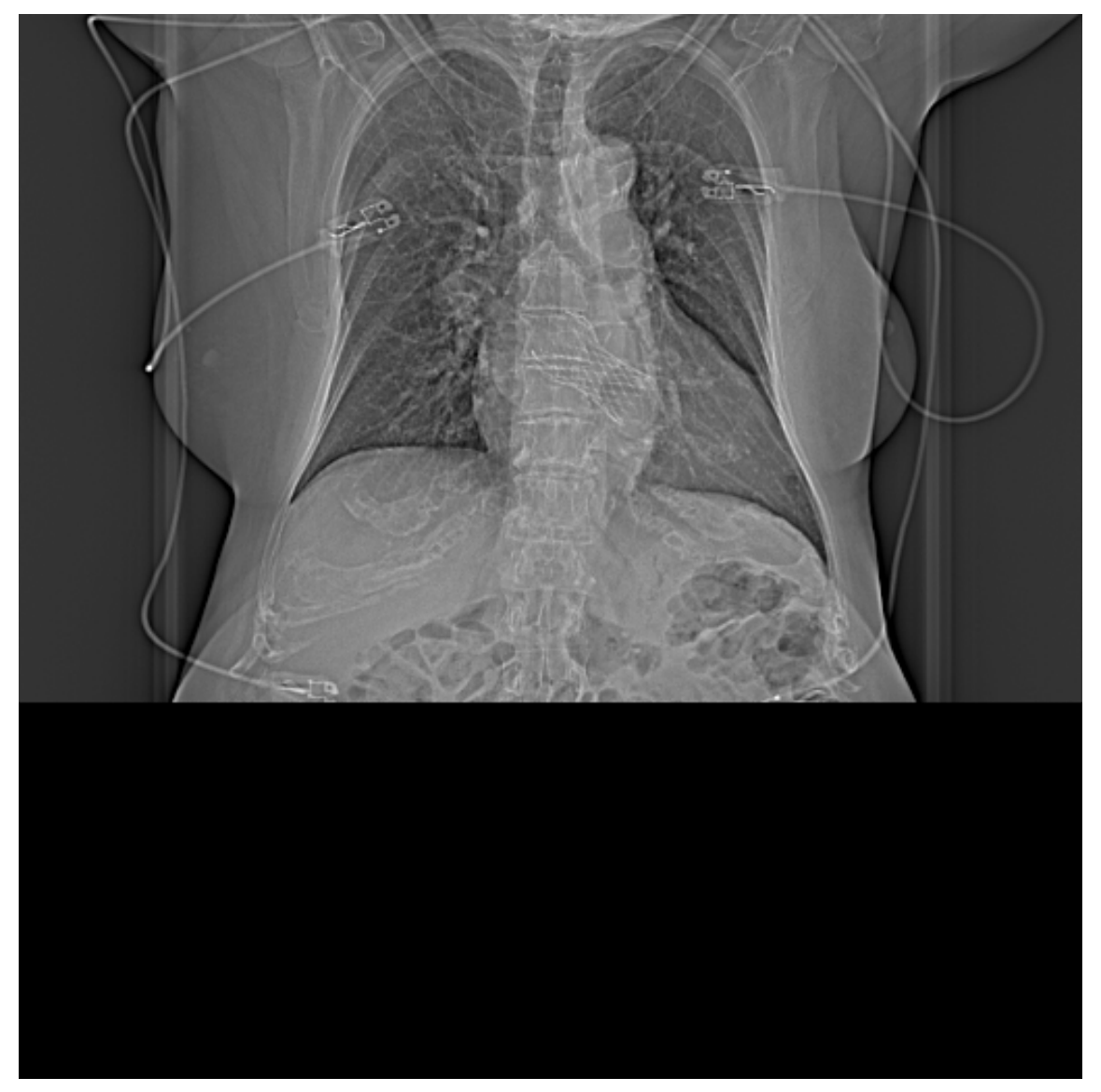

